\title{
Artículo
}

\section{Secuencia de intubación rápida en el Servicio de Emergencias: una revision bibliográfica} Rapid-sequence intubation in the Emergency Department: a review

Autores

Dra. Mónica Alvarado Ferllini

Médico General, graduada dela Universidad de Costa Rica. Médico independiente, San José, Costa Rica.

Correspondencia:monicaferllini@hotmail.com

\section{Resumen}

La secuencia de intubación rápida se define como la administración de un agente hipnótico y un relajante neuromuscular, junto con otros procedimientos que inducen inconsciencia $y$ parálisis muscular para facilitar la intubación traqueal rápida y minimizar el riesgo de aspiración, en pacientes que no logran mantener la vía aérea protegida. Una de sus contraindicaciones relativas es la ausencia de entrenamiento, aunque todo el personal médico debe estar capacitado para ejecutar el procedimiento. La técnica se compone de siete pasos que deben dominar todos los médicos, en especial, aquellos que laboran en un servicio de emergencias, donde esta secuencia es parte fundamental del manejo avanzado de la vía aérea para disminuir la morbimortalidad.
Palabras clave

Intubación Intratraqueal, Urgencias Médicas, Manejo de la Vía Aérea, Anestesiología.

\section{Abstract}

Rapid-sequence intubation is defined as the administration of a hypnotic agent and a neuromuscular relaxant, along with other procedures that induce unconsciousness and muscle paralysis to facilitate rapid tracheal intubation and minimize the risk of aspiration in patients who fail to maintain a protected airway. One of their relative contraindications is the absence of training, although all medical personnel should be trained to perform the procedure. The technique is composed of seven steps that must be mastered by all physicians, 
especially those working in an emergency department, where this sequence is a fundamental part of advanced airway management to decrease morbimortality.

\section{Keywords}

Intubation, Intratracheal, Emergencies, Airway Management, Anesthesiology.

\section{Introducción}

A través de la historia, se ha señalado que la primera intubación de tráquea en humanos fue demostrada por Curry en 1792. En 1943, Sir Robert Macintosh conceptualizó la intubación como la inserción de un tubo en el interior de la tráquea con el fin de ventilar, oxigenar, aspirar y proteger el árbol bronquial (1).

Posteriormente, en julio de 1970, Stept y Safar publicaron su protocolo de inducción-intubación para prevenir la aspiración gástrica, que contemplaba la administración de tiopental sódico y succinilcolina, con lo cual obtenían condiciones de intubación en menos de 1 minuto (2).

En la actualidad, se sabe que el manejo de la vía aérea es una de las prioridades en un paciente crítico, pero también es uno de los mayores desafíos al que se exponen los médicos, tomando en cuenta que el resultado final depende de las características del paciente, los equipos y la destreza del operador, lo cual puede ser un determinante de la morbilidad y mortalidad $(3,4)$.

Por ello, es vital lograr el control de la vía aérea (5), lo cual implica dominar la secuencia de intubación rápida (SIR), puesto que es el procedimiento estándar para el manejo avanzado de la vía aérea en los servicios de urgencias (6). Debido a lo anterior, el objetivo de la presente revisión es describir las indicaciones, contraindicaciones, la técnica correcta de la secuencia de intubación rápida aplicable a un servicio de emergencias, además de algunas de sus complicaciones inmediatas.

\section{Definición}

La SIR se define como la administración de un agente hipnótico y un relajante neuromuscular de forma virtualmente simultánea, así como de otros procedimientos que inducen inconsciencia y parálisis muscular para facilitar la intubación traqueal rápida. El propósito primordial es minimizar el riesgo de aspiración (7, 8), especialmente, en individuos con una pérdida inminente de las vías respiratorias o con graves problemas de intercambio de gases que requieren ventilación mecánica (9).

\section{Anatomía de las vías aéreas superiores}

La vía aérea en los seres humanos presenta dos aperturas, una nasal y una bucal, que se unen en la parte posterior con la faringe, la cual es una estructura fibromuscular extendida desde la base del cráneo hasta el cartílago cricoides (1). A su vez, la faringe se divide en: nasofaringe, que comunica con la fosa nasal, orofaringe, que comunica con la cavidad oral y laringofaringe o hipofaringe (10). En la base de la lengua, la epiglotis hace una separación funcional entre bucofaringe y laringofaringe y evita la aspiración al cubrir la glotis (la entrada de la laringe) durante la deglución (1).

La laringe está constituida por un esqueleto cartilaginoso al cual se unen estructuras musculares. Se encuentra situada en la porción anterior del cuello y mide aproximadamente 5 $\mathrm{cm}$ de longitud, si bien es más corta y cefálica en las mujeres y en los niños. Su estructura consta de nueve cartílagos: 1 cricoides, 1 tiroides, 1 epiglotis, 2 aritenoides, 2 corniculados o de Santorini y 2 cuneiformes o de Wrisberg (10). En la figura 1 se observa la anatomía normal de la laringe durante inspiración (11): 


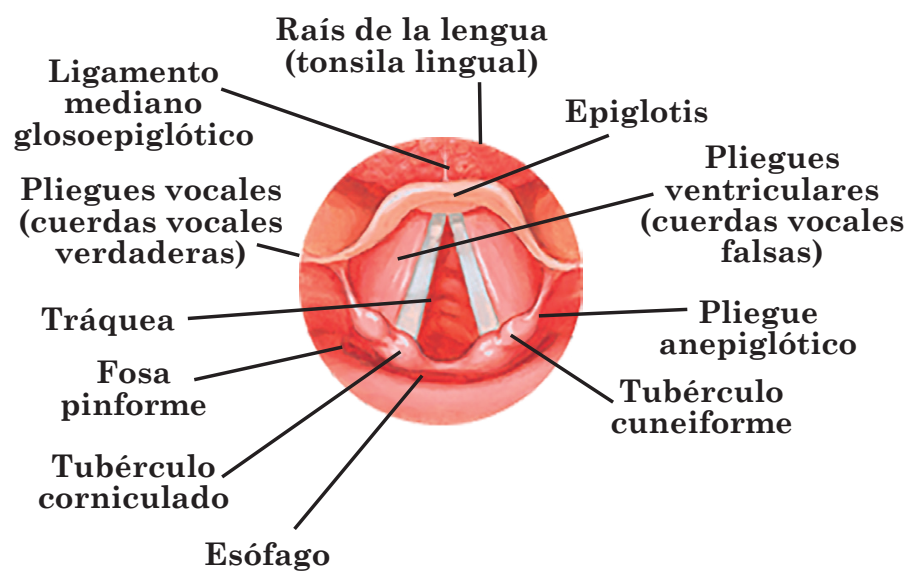

Figura 1. Anatomía normal de la laringe durante inspiración. Adaptado al español de: Netter FH. Atlas of Human Anatomy. 6th ed. Philadelphia: Saunders Elsevier; 2014.

\section{Indicaciones de SIR}

En general, los pacientes en los que se sospecha que no podrán mantener la vía aérea protegida deben ser intubados (3). Algunas indicaciones son:

- Glasgow igual o menor a 8 (5).

- Hematoma sofocante en cuello (3).

- Obstrucción de la vía aérea (3).

- Trauma de la vía aérea o maxilofacial (3).

- Paciente agitado que requiere sedación (3).

- Trauma torácico con hipotensión (3).

- Hipoxia posreanimación (3).

- Paro cardiaco (3).

- Edema de la vía aérea (quemaduras, anafilaxia) (5).

- Quemaduras extensas (3).

- Trauma raquimedular cervical (3).

- Choque severo (3).

- Insuficiencia respiratoria $(>30 \quad 0 \quad<10$ respiraciones por minuto) (5).

- Elevación de la PaCO2 (>40 mm Hg) con caída del pH (5).

- $\mathrm{PaO} 2<50 \mathrm{~mm} \mathrm{Hg}$ con mascarilla de no reinhalación, principalmente, cuando asocia signos clínicos de hipoxemia (agitación, confusión, "pelea" contra la mascarilla de oxígeno) (5).

- Incapacidad para mantener la vía aérea permeable (3).

\section{Contraindicaciones y precauciones}

Las contraindicaciones de la SIR son relativas (8). Algunas de estas son:

- Ausencia de entrenamiento en la técnica, aunque en condiciones de emergencia y ausencia de un experto, puede ser efectuada por médicos con el conocimiento teórico (5).

- Proximidad estrecha al centro receptor (5).

- Anticipación de la dificultad de intubación, en particular, si se considera que la oxigenación de rescate es difícil o imposible (8).

- Cuando un paciente no puede tolerar la apnea (por ejemplo, cuando hay hipoxemia profunda o acidosis metabólica), el bloqueo neuromuscular puede ser arriesgado y se prefiere un enfoque de intubación "despierto", es decir, el uso de anestesia tópica y sedación ligera (8).

- Del mismo modo, según las circunstancias clínicas, se pueden seleccionar o evitar determinados agentes sedantes o bloqueantes neuromusculares (8).

\section{Descripción de la secuencia de intubación rápida}

El orden de la SIR se resume en las "siete P", a continuación, se muestran estos pasos y el tiempo ideal en el que deben ejecutarse (12):

1) Preparación: 10 minutos antes de la intubación.

2) Preoxigenación: 5 minutos antes de la intubación.

3) Pretratamiento: 3 minutos antes de la intubación.

4) Parálisis con inducción.

5) Posición y protección: 30 segundos después de la inducción.

6) Intubación (Placement): 45 segundos después de la inducción.

7) Postintubación: 60 segundos después de la inducción. 


\section{1) Preparación:}

En esta etapa se organiza el equipo de salud, se asignan las funciones que desempeñará cada profesional, se preparan los equipos de monitorización y reanimación, además de los medicamentos (6). Asimismo, en esta fase deben ser evaluadas las vías respiratorias del paciente para detectar posibles dificultades (8) y contemplar un plan alternativo de intubación (6). La mnemotecnia "SOFAME" es una manera de recordar fácilmente los aspectos más relevantes de una preparación exitosa (6).

S: Succión. Debe ser realizada por el operador que realiza la laringoscopia, por lo que el equipo de succión debe estar al lado derecho de este (6). O: Oxígeno. Se refiere a la administración de oxígeno mediante mascarilla de alto flujo, nasocánula o el dispositivo que esté disponible; lo importarte es asegurarse de que estará disponible en el momento de la intubación (6).

F: Fármacos. Se deben incluir los medicamentos para el pretratamiento (lidocaína, fentanilo o atropina), inducción (midazolam, fentanilo, tiopental, etomidato o ketamina), parálisis (succinilcolina o rocuronio), antiarrítmicos (amiodarona, adenosina y sulfato de magnesio) y vasopresores (adrenalina, norepinefrina y vasopresina) $(6,13)$.

A: Vía aérea. En esta etapa se recomienda evaluar la vía aérea, las necesidades de posicionamiento del paciente e intentar predecir el riesgo de una vía aérea difícil (6). Existen 2 mnemotecnias para ayudar a prever esto: "LEMON" y "MOANS".

\section{LEMON:}

L: Look externally (mirar externamente). Examen corto dirigido al cuello, la boca y la vía aérea interna (7).

E: Evaluate (evaluar). Aplicación de la regla 3-3-2: apertura oral con introducción de 3 dedos entre los dientes inferiores y superiores, 3 dedos entre el mentón y el inicio del cuello y 2 dedos entre el cartílago tiroideo y el suelo de la mandíbula, que son las dimensiones idóneas para la intubación (7).
M: Mallampati. Tras la visualización de las estructuras anatómicas faríngeas, se clasifica en cuatro grados: I (visibilidad del paladar blando, úvula y pilares amigdalinos), II (visibilidad del paladar blando y úvula), III (visibilidad del paladar blando y base de la úvula) y IV (imposibilidad para ver el paladar blando) (14). Los grados III y IV predicen dificultad para la intubación (7).

O: Obstruction of the airway (obstrucción de la vía aérea).

N: Neck mobility (movilidad del cuello). Limitación de la flexoextensión (7).

\section{MOANS:}

M: Mask seal (sello de la máscara). Dificultad o compromiso (15).

O: Obstruction/Obesity (obstrucción/obesidad). Especialmente, obstrucción supraglótica (15).

A: Advanced age (edad avanzada). Mayor riesgo en $>55$ años (15).

$\mathrm{N}$ : No teeth ( $\sin$ dientes). Interfiere también con el sello de la máscara (15).

S: Stiffness/resistance to ventilation (rigidez/resistencia a la ventilación). Enfermedades pulmonares, embarazo de término, entre otros (15).

M: Monitoreo. La monitorización básica durante el procedimiento incluye la medición de la frecuencia cardiaca, presión arterial, frecuencia respiratoria, saturación de oxígeno mediante oximetría de pulso, monitoreo de ritmo cardiaco mediante e, idealmente, capnografía (6).

E: Equipo. Se recomienda revisar y preparar el equipo necesario, incluyendo al personal médico, de enfermería y auxiliares (6). Los instrumentos necesarios son los siguientes:

- Laringoscopio con fuente de luz óptima y valvas de diferentes tamaños $(13,3)$.

- Tubos endotraqueales (TET) de diferentes tamaños (13). En mujeres adultas, se recomienda un tubo número 6.5 a 7.5; en varones, 7.5 a 8.5 , aunque varía según la estatura y composición corporal (3). 
- Guía maleable para tubo endotraqueal y lubricante (13).

- Máscara con bolsa y válvula de no reinhalación (13).

- Cánulas orofaríngeas de distintos tamaños (13).

- Equipo de succión y sus respectivas sondas (13).

- Jeringa de $10 \mathrm{~cm}$ adaptada al tutor del neumotaponador del tubo (3).

- Fuente de oxígeno (13).

- Equipo alternativo para situación de vía aérea difícil con intubación fallida (máscara laríngea, equipo de cricotiroidotomía) (13).

- Fijador comercial del tubo o, en su defecto, microporo, fixu-mull o esparadrapo (3).

Carro de paro (3).

\section{2) Preoxigenación:}

Se utiliza para mejorar la saturación de oxihemoglobina del paciente, ya que el alto flujo desplaza el nitrógeno pulmonar y lo sustituye por oxígeno, lo que convierte la capacidad residual funcional de los pulmones en una reserva de oxígeno. Este efecto permite que los pacientes toleren un periodo de apnea más largo sin desaturación clínica. Es esencial para la seguridad del paciente y para evitar ventilaciones asistidas después de la parálisis y hasta la colocación del TET (12).

La preoxigenación se logra administrando alto flujo de oxígeno por 3-5 minutos mediante una máscara de no reinhalación o realizando 8 respiraciones máximas durante 1 minuto, espontáneas 0 asistidas. La meta es lograr una saturación $>93 \%$, idealmente, $100 \%$ (12). No obstante, se debe considerar que los niños y los pacientes con elevación del diafragma (por embarazo, obesidad, aumento de la tensión intraabdominal) tienen menor capacidad funcional residual, por lo que en ellos la preoxigenación será menos efectiva (6).

\section{3) Pretratamiento:}

Consiste en la administración de fármacos antes de proceder a la inducción-relajación con el fin de mitigar los efectos adversos secundarios a la intubación orotraqueal (hipotensión, bradicardia o taquicardia, aumento de presión intracraneal y resistencia de la vía aérea), si bien no se utilizan por regla general (7).

La mnemotecnia "LOAD" se refiere al empleo de los siguientes fármacos: lidocaína, opioides, atropina y dosis desfasciculantes de succinilcolina, según corresponda (6).

- L: Lidocaína. Se indica en pacientes con hipertensión intracraneana o aumento de la reactividad bronquial, ya que disminuye la respuesta a la manipulación de la vía aérea. Dosis recomendada: $1.5 \mathrm{mg} / \mathrm{kg}$ de peso IV $(6,7)$.

- O: Opioides. El fentanilo es un opioide de vida media corta y de rápido inicio de acción que disminuye la respuesta simpática (taquicardia e hipertensión arterial) durante la manipulación de la vía aérea. Asimismo, disminuye la presión intracraneal en pacientes con trauma de cráneo y signos de hipertensión intracraneal. Dosis recomendada: $2-3 \mathrm{mcg} / \mathrm{kg}$ de peso IV en bolo lento (30-60 segundos) $(6,7)$.

- A: Atropina. Se recomienda más en niños o en caso de dosis repetidas de succinilcolina en adultos, para prevenir bradicardia e hipotensión. Dosis recomendada: 0.02 $\mathrm{mg} / \mathrm{kg}$ de peso IV en bolo rápido (mínimo 0.1 mg, máximo $0.5 \mathrm{mg})(5,7)$.

- D: Dosis desfasciculantes de succinilcolina. Ya no se recomienda su uso, debido a sus efectos adversos y que se desconoce el beneficio real en disminuir las fasciculaciones (6).

De los tres fármacos recomendados, en la Caja Costarricense de Seguro Social (CCSS) se cuenta con todos: atropina $0.5 \mathrm{mg} / \mathrm{ml}$ en ampollas de $1 \mathrm{ml}$, lidocaína $20 \mathrm{mg} / \mathrm{ml}$ en ampollas de 5 o $10 \mathrm{ml}$ y fentanilo $0.05 \mathrm{mg} / \mathrm{ml}$ en ampollas de $2 \mathrm{ml}$, este último se recomienda diluirlo en $8 \mathrm{ml}$ de $\mathrm{NaCl}$ 0.9\%, aunque se encuentra restringido para Anestesiología y Cuidados Intensivos (16). 
4) Parálisis con inducción:

Se administra de manera casi simultánea un agente inductor de acción rápida y un bloqueador neuromuscular para producir un estado de sedación profunda y relajación muscular rápidamente (12). En la actualidad, los sedantes más utilizados son: etomidato, ketamina, midazolam y propofol (ver cuadro 1) (7). Se podría utilizar tiopental, pero este se evita en el ámbito de la medicina de emergencias durante la SIR, dado que se asocia a deterioro agudo del equilibrio hemodinámico (13) y puede exacerbar el broncoespasmo (12).
- Etomidato: agente inductor de efecto hipnótico más empleado, no afecta la hemodinamia (6).

- Ketamina: es el único inductor que tiene efectos analgésicos (6).

- Propofol: sedante-hipnótico de acción ultracorta. Su principal ventaja radica en su rápido inicio de acción y su corta duración (6).

- Benzodiazepinas: el midazolam es el más empleado de este grupo, por su efecto rápido y corta duración (6).

Cuadro 1. Características de medicamentos sedantes.

\begin{tabular}{|l|c|c|c|c|}
\hline & Etomidato & Ketamina & Midazolam & Propofol \\
\hline $\begin{array}{l}\text { Dosis } \\
\text { (mg/kg) }\end{array}$ & 0.3 & 1.5 & 0.3 & 1.5 \\
\hline Inicio (seg) & $15-45$ & $50-60$ & $60-90$ & $15-45$ \\
\hline $\begin{array}{l}\text { Vida media } \\
\text { (min) }\end{array}$ & $2-4$ & $11-17$ & $7-15$ & $1-3$ \\
\hline $\begin{array}{l}\text { Duración } \\
\text { (min) }\end{array}$ & $3-12$ & $10-20$ & $15-30$ & $5-10$ \\
\hline Beneficios & $\begin{array}{l}\text { Excelente } \\
\text { sedante } \\
\text { con poca } \\
\text { hipotensión }\end{array}$ & $\begin{array}{l}\text { Simula } \\
\text { liberación de } \\
\text { catecolaminas }\end{array}$ & Broncodilatador & Broncodilatador \\
\hline Precauciones & $\begin{array}{l}\text { Supresión } \\
\text { de producción } \\
\text { adrenal de } \\
\text { cortisol }\end{array}$ & $\begin{array}{l}\text { Controversial } \\
\text { si presión } \\
\text { intracreaneal } \\
\text { elevada }\end{array}$ & $\begin{array}{l}\text { Depresión } \\
\text { miocárdica } \\
\text { dosis } \\
\text { dependiente }\end{array}$ & $\begin{array}{l}\text { Hipotensión } \\
\text { dosis } \\
\text { dependiente }\end{array}$ \\
\hline
\end{tabular}

Fuente: $(6,12)$ 
A nivel de la CCSS, de estos cuatro fármacos se cuenta con: ketamina $10 \mathrm{mg} / \mathrm{ml}$ en frascos de 20 $\mathrm{ml}$, propofol $10 \mathrm{mg} / \mathrm{ml}$ en frascos de $20 \mathrm{ml}$ y midazolam $5 \mathrm{mg} / \mathrm{ml}$ en ampollas de $3 \mathrm{ml}$ (16). El más disponible y más utilizado es el midazolam, que se recomienda diluirlo en $12 \mathrm{ml}$ de $\mathrm{NaCl}$ 0.9\% para alcanzar una solución 1:1 (5).

Con respecto a los agentes para bloqueo neuromuscular, existen dos categorías según su mecanismo de acción: agentes despolarizantes (succinilcolina) y agentes no despolarizantes (vecuronio y rocuronio). Los fármacos despolarizantes imitan la acción de la acetilcolina en el receptor nicotínico de la placa motora, lo que produce despolarización sostenida que impide la contracción, mientras que los agentes no despolarizantes actúan mediante inhibición competitiva.

Los más utilizados son la succinilcolina, el rocuronio y, con menor frecuencia, el vecuronio (ver cuadro 3) (6). Sin embargo, en la CCSS solo está disponible la succinilcolina $50 \mathrm{mg} / \mathrm{ml}$ en frascos de $10 \mathrm{ml}$ (16), que se recomienda diluir en $10 \mathrm{ml}$ de $\mathrm{NaCl} 0.9 \%$ (5).

Una ventaja del rocuronio es que ahora existe el sugammadex, una ciclodextrina que encapsula los relajantes musculares no despolarizantes aminoesteroideos, como el rocuronio. Su inicio de acción es inmediato y la recuperación del 90\% del tren de cuatro es alrededor de los $3 \mathrm{~min}$ con una dosis de $16 \mathrm{mg} / \mathrm{kg}$, lo que lo convierte en una gran alternativa para el uso en intubación de secuencia rápida (3).

Seguidamente, se indican las principales características de la succinilcolina y rocuronio $(3,6,7)$ :
Succinilcolina:

- Dosis: $1.5 \mathrm{mg} / \mathrm{kg}$.

- Inicio: 45 segundos.

- Duración: 6-10 minutos.

- Efectos adversos: fasciculaciones, hipercalemia, bradicardia, hipertermia maligna, bloqueo neuromuscular prolongado.

- Contraindicaciones: antecedentes que predispongan a sus efectos adversos (esclerosis múltiple, sepsis, miopatías, entre otros).

\section{Rocuronio:}

- Dosis: $1.2 \mathrm{mg} / \mathrm{kg}$.

- Inicio: 60-75 segundos.

- Duración: 40-60 minutos.

- Efectos adversos: hipersensibilidad al fármaco, leve hipotensión, taquicardia (raros).

- Precauciones: en insuficiencia hepática.

\section{5) Posición y protección:}

El posicionamiento del cuello y la cabeza del paciente es clave para una mejor visión de las cuerdas. Se deben alinear los tres ejes: oral, faríngeo y laríngeo (6). Esto se logra con la llamada posición de olfateo, en la que se hiperextiende la cabeza con respecto al cuello y este se flexiona con respecto al tronco (7).

En cuanto a la protección de la vía aérea, se refiere al uso de la maniobra de Sellick para prevenir la distensión gástrica y la aspiración de contenido gástrico durante el procedimiento, esta técnica involucra comprimir el cartílago cricoides en sentido anteroposterior con los dedos índice y pulgar (6).

\section{6) Intubación (Placement):}

El procedimiento se realiza de la siguiente forma: se abre la boca y se introduce la hoja del laringoscopio en dirección opuesta a la mano con que se sostiene el laringoscopio, buscando el pilar anterior de la amígdala y luego girando la punta de la hoja hacia el centro, para tratar de 
mantener la lengua del paciente por fuera del campo visual, haciendo una fuerza sostenida en dirección hacia arriba y un poco hacia adelante hasta exponer las cuerdas vocales (13).

En el caso de los adultos, el uso de la hoja curva del laringoscopio de Macintosh es más frecuente, con la cual se coloca la punta de la hoja en la valécula o surco glosoepiglótico y se hace visible la epiglotis, que se levanta para exponer la glotis. En los pacientes pediátricos, es más útil la hoja recta del laringoscopio de Miller, que mejora la exposición de las estructuras glóticas, al levantar con ella la epiglotis directamente. Durante esta maniobra, se debe evitar un movimiento de palanca, intentando levantar la punta de la hoja del laringoscopio (13).

Para facilitar la exposición de la glotis, se describió la maniobra BURP, acrónimo en inglés que describe el desplazamiento que se debe realizar durante la laringoscopia presionando el cartílago tiroides hacia atrás, arriba y a la derecha (backward, upward, and rightward pressure), con el objetivo de mejorar la visibilidad de la laringe (13). Una vez que se visualiza la glotis, el clínico coloca el TET entre las cuerdas vocales, infla el manguito, retira la guía y confirma la colocación (8).

\section{7) Postintubación:}

Los cuidados postintubación inician inmediatamente después del procedimiento. Se debe comprobar que el TET esté bien colocado, lo cual clásicamente se ha logrado con la observación del paso del tubo a través de las cuerdas vocales, la auscultación pulmonar y del epigastrio, la elevación del tórax durante las primeras ventilaciones a presión positiva, la visualización de condensación de vapor de agua en el TET, la capnografía y el estudio radiológico $(17,18)$. No obstante, más recientemente, se está utilizando también la ecografía, cuyo único inconveniente es que es operador dependiente y que el clínico que la realice debe tener pericia (18).
Asimismo, en esta última fase, se trata la enfermedad base, se administra una adecuada sedación, analgesia y relajación de mantenimiento, se ajustan los parámetros de la ventilación mecánica, se monitorizan de forma exhaustiva los signos vitales y se realiza una radiografía de tórax para diagnosticar de forma rápida algunas complicaciones (7).

\section{Complicaciones agudas}

Las complicaciones se pueden dividir en:

- Mayores: aumentan la morbimortalidad inmediata. Algunos ejemplos son: neumotórax y enfisema por barotrauma, aparición de déficit neurológico por una lesión cervical que no existía previamente, intubación esofágica no detectada, traumatismo de la vía aérea con hemorragia, broncoaspiración, paro cardiorrespiratorio, bradicardia (5).

- Menores: aumentan morbilidad, mas no mortalidad. Algunas son las siguientes: atelectasia lobar, intubación del bronquio principal derecho, tiempo de intubación prolongadado (>30 segundos), TET desplazado, TET dañado, trauma dental (5).

\section{Conclusiones}

La secuencia de intubación rápida implica una serie de procedimientos que facilitan la intubación traqueal expedita para disminuir el riesgo de aspiración. Esta técnica es clave para el manejo avanzado de la vía aérea en los servicios de urgencias y está indicada en pacientes en los cuales se sospecha que no podrán mantener la vía aérea protegida. No obstante, existen algunas contraindicaciones relativas para su ejecución, como la falta de experiencia.

La secuencia se resume en siete pasos fundamentales: preparación, preoxigenación, pretratamiento, parálisis e inducción, posición y protección, intubación y cuidados postintubación. Estas etapas deben ser conocidas por todo el personal médico para que, 
en caso de que se requiera realizar una intubación en el servicio de emergencias, se puede realizar correctamente, con la finalidad de disminuir morbimortalidad.

\section{Referencias}

1. Dávila E, López R, Márquez F, Hernández CM. Intubación de secuencia rápida. Medisur. 2015 Set; 13(4): 533-540.

2. Poveda R, Dueñas C, Ortiz G. Secuencia rápida de intubación en cuidados intensivos. Rev Colomb Anestesiol. 2013; 41(1): 24-33.

3. Almarales JR, Saavedra MA, Salcedo O, Romano DW, Morales JF, Quijano CA, et al. Intubación de secuencia rápida para intubación orotraqueal en Urgencias. Repert Med Cir. 2016; 25(4): 210-218.

4. Coloma R, Álvarez JP. Manejo avanzado de la vía aérea. Rev Med Clin Condes. 2011; 22(3): 270-279.

5. Arias WA, Ramírez JL. Secuencia rápida de intubación en el primer nivel de atención. Revista Médica de Costa Rica y Centroamérica. 2013; LXX(608): 671-678.

6. Maluenda F, Aguilera P, Kripper C, Navea O, Basaure C, Saldías F. Secuencia rápida de intubación en el Servicio de Urgencia. Revista Chilena de Medicina Intensiva. 2015; 30(1): 23-32.

7. Parrilla FM, Aguilar I, Cárdenas D, López L, Cárdenas A. Secuencia de intubación rápida. Emergencias. 2012; 24: 397-409.

8. Brown C, Sakles JC. Rapid sequence intubation for adults outside the operating room. UpToDate [Internet]. 2019 Set 24 [citado 2020 Mar 13]. Disponible en: https://www.uptodate.com
Stollings JL, Diedrich DA, Oyen LJ, Brown DR. Rapid-Sequence Intubation: A Review of the Process and Considerations When Choosing Medications. Annals of Pharmacotherapy. 2014; 48(1): 62-76.

García HF, Gutiérrez SE. Aspectos básicos del manejo de la vía aérea: anatomía y fisiología. Revista Mexicana de Anestesiología. 2015 Abr-Jun; 38(2): 98-107.

Netter FH. Atlas of Human Anatomy. 6th ed. Philadelphia: Saunders Elsevier; 2014.

Archila A. Intubación de secuencia rápida en patologías selectas. R Int Med Emergencias. 2015; 1(2): 31-35.

Pérez P, Moreno A, Gempeler FE. Guía para la secuencia de inducción e intubación rápida en el servicio de emergencias. Univ Méd. 2013 Abr-Jun; 54(2): 175-198.

Echevarría AT, Autié Y, Hernández K, Díaz C, Sirvent Y. Pruebas predictivas para la evaluación de la vía aérea en el paciente quirúrgico. Rev Cuba Anestesiol Reanim. 2010 Set-Dic; 9(3): 175-185.

Walls RM, Hockerberg RS, Gausche M, Bakes K, Baren JM, Erickson TB, et al, editors. Rosen's Emergency Medicine Concepts and Clinical Practice. Philadelphia: Elsevier; 2018.

Caja Costarricense de Seguro Social. Lista oficial de medicamentos y normativa. Costa Rica: Caja Costarricense de Seguro Social; 2014.

Chiappero G, Criado F. Confirmación de la intubación orotraqueal. Medicina Intensiva. 2010; 18(2): 79-80.

Díaz HR. Comprobación de la posición del tubo endotraqueal mediante ecografía clínica. Gaceta Médica Espirituana. 2017; 19(1): 1-6. 